\title{
Waveguiding microstructures in Nd:YAG with cladding and inner dual-line configuration produced by femtosecond laser inscription
}

\author{
Hongliang Liu ${ }^{a}$, Javier R. Vázquez de Aldana ${ }^{b}$, Blanca del Rosal Rabes ${ }^{c}$, Feng Chen ${ }^{\mathrm{a}, *}$ \\ a School of Physics, State Key Laboratory of Crystal Materials and Key Laboratory of Particle Physics and Particle Irradiation (MOE), Shandong University, Jinan 250100, China \\ ${ }^{\mathrm{b}}$ Laser Microprocessing Group, Universidad de Salamanca, Salamanca 37008, Spain \\ ${ }^{\text {c } F l u o r e s c e n c e ~ I m a g i n g ~ G r o u p, ~ D e p a r t a m e n t o ~ d e ~ F i ́ s i c a ~ d e ~ M a t e r i a l e s, ~ F a c u l t a d ~ d e ~ C i e n c i a s, ~ U n i v e r s i d a d ~ A u t o ́ n o m a ~ d e ~ M a d r i d, ~ M a d r i d ~ 28049, ~ S p a i n ~}$
}

\section{A R T I C L E I N F O}

\section{Article history:}

Received 21 July 2014

Received in revised form 8 November 2014

Accepted 9 November 2014

Available online 25 November 2014

\section{Keywords:}

Femtosecond laser inscription

Optical waveguides

Waveguide lasers

Nd:YAG crystal

\begin{abstract}
A B S T R A C T
We demonstrate a new design of waveguiding microstructure, which contains both cladding and inner dual-line configurations, in Nd:YAG laser crystals inscribed by femtosecond laser processing. Based on this prototype, a few "cladding + dual-line" hybrid structures with different parameters have been successfully manufactured in Nd:YAG. The waveguide lasing at $1.06 \mu \mathrm{m}$ has been realized in the hybrid structures under 808-nm optical pump. Compared to the single dual-line waveguides, the dual-line core in hybrid configuration benefits from the large-area pump from the external cladding, reaching an enhancement of $26 \%$ on the maximum output power and of $24 \%$ on the slope efficiency of the waveguide lasing. In addition, the symmetric stress field of external cladding structure produces anisotropic stress field in the inner cores, supporting guidance along both TE and TM polarizations, thus differing significantly from the single dual-line waveguides.
\end{abstract}

(ㄷ) 2014 Elsevier B.V. All rights reserved.

\section{Introduction}

The femtosecond (fs) laser inscription (FLI) has become a powerful technique to produce micro and submicrometric structures in transparent dielectric materials [1,2]. In crystalline materials, the fs laser pulses create either positive or negative refractive index changes through modification of diverse parameters, such as lattice distortion, stress effects, or volume expansion [3-6]. During the processing, the energy of the fs pulses is deposited in the substrates by the avalanche ionization and nonlinear absorption process, such as two-photon or multiphoton absorption without a fast heat transfer $[7,8]$. FLI has rapidly become a widespread method to produce waveguiding microstructures in various optical materials since the pioneering work of Davis et al. [9] in 1996. As of yet, waveguide lasers with enhanced efficiencies and reduced lasing thresholds have been achieved in the FLI-produced waveguides $[10,11]$.

In the cubic Nd:YAG crystals, FLI usually causes negative refractive index changes in the fs-laser-induced tracks, and the waveguiding core is located in the vicinity of the damage lines. The well-known FLI-manufactured dual-line waveguides (usually called Type II configuration, containing two parallel lines) have

\footnotetext{
* Corresponding author. Tel.: +86 531 88363007; fax: +86 53188363350 . E-mail address: drfchen@sdu.edu.cn (F. Chen).
}

been extensively investigated by a few groups, and efficient lasing has been realized in the dual-line platforms [12-17]. However, the dual-line waveguides only guide TM-polarized light, possibly due to the stress field modifications along the vertical dimension at transverse cross section, which may limit the polarization-free applications. Recently, depressed cladding waveguides (so-called Type III) containing a number of damage tracks with reduced indices [18-21] support guidance in the track-surrounded cores along any transverse polarization. In addition, benefiting from the large scale of the diameters of the cladding structures, a more efficient pump and a flexible integration of optical fibers may be achieved [22-28]. Nevertheless, the shortcoming of the cladding waveguides is the relatively poor guided modes (mostly highly multiple-mode for large scale cladding structures).

In this work, we propose a new design, which contains an outer cladding and an inner dual-line structure, for FLI processed Nd:YAG crystals. This "cladding + dual-line" structure has similar feature with our former "double-cladding" configuration [26], which both resemble to the configuration of the well-known double-clad fibers and benefit from large-cross-section pump of outer cladding) [29]. The confocal micro-photoluminescence spectroscopy was utilized to investigate the details of the FLI modification in the "cladding + dual-line" structures. Waveguiding lasers with enhanced performances at both TE and TM polarizations at $1.06 \mu \mathrm{m}$ in the inner dual-line cores in the hybrid structures were further realized. 


\section{Experiments in details}

The Nd:YAG crystal (doped by 1 at.\% $\mathrm{Nd}^{3+}$ ions) used in this work was optically polished and cut into a sample of dimensions $8 \mathrm{~mm} \times 10 \mathrm{~mm} \times 2 \mathrm{~mm}$. The "cladding + dual-line" hybrid structures were produced at the laser facility of Universidad de Salamanca, Spain. The cross section of the resulting structures (labeled by W1-W6) in the Nd:YAG crystal can be seen in Fig. 1(a)-(f). During the micromachining process, an amplified Ti:Sapphire laser system was used to generate linearly-polarized $120 \mathrm{fs}$ pulses at a central wavelength of $800 \mathrm{~nm}$ (with $1 \mathrm{kHz}$ repetition rate). The pulse energy used to micro-process the sample was set with a calibrated neutral density filter, a half-wave plate and a linear polarizer. The sample was placed in a computer controlled motorized 3-axes stage. The beam was focused through the largest sample surface $(8 \mathrm{~mm} \times 10 \mathrm{~mm}$ ) at a depth of $150 \mu \mathrm{m}$ through a microscope objective (Leica $40 \times$, N.A. $=0.65$ ) with a pulse energy of $2.52 \mu \mathrm{J}$. During the irradiation, the sample was moved at a constant speed of $500 \mu \mathrm{m} / \mathrm{s}$ in the direction perpendicular to both the fs-laser propagation as the polarization direction to fabricate depressed cladding waveguides, and at a constant speed of $50 \mu \mathrm{m} / \mathrm{s}$ to construct dualline waveguides. Meanwhile, the pulse propagation was carefully aligned with the $10-\mathrm{mm}$ long edge of the sample, thus producing a damage track along the sample. Many parallel scans (with $\sim 3 \mu \mathrm{m}$ separation between adjacent damage tracks) were performed at different depths of the sample (from bottom to top in order to avoid the shielding of the incident pulses by the previously written damage tracks) to inscribe hybrid waveguides, which consist of larger-size cladding structures, $100 \mu \mathrm{m}$ and $150 \mu \mathrm{m}$ in diameter, and dual-line structures at the center of the claddings with a separation between lines of $15 \mu \mathrm{m}$ and $20 \mu \mathrm{m}$, respectively. For the sake of comparison, single dual-line waveguides with the same dimension were manufactured in the same sample.

The homemade confocal microscope used for the micro-fluorescence investigation of the waveguides is quite similar to that (Olympus BX-41 fiber-coupled confocal microscope) used for the experimental setup depicted in a previous work [18]. The 488-nm continuous wave (cw) diode laser, as the optical excitation source, was focused in the sample by a $50 \times$ microscope objective (N.A. $\sim 0.55$ ), thus yielding a spot with a diameter of approximately
$0.53 \mu \mathrm{m}$. The subsequent emission of $\mathrm{Nd}^{3+}$ ions was collected by the same microscope objective and, after passing through filters, lenses and pinholes, was analyzed by a high resolution spectrometer. The software LabSpec ${ }^{\circledR}$ and WSxM ${ }^{\circledR}$ [30] were used to analyze and fit the spectra and process the fluorescence images in terms of the spectral intensity, energy shift and width of the fluorescence lines.

End-pumped arrangement was used to perform the properties of the cw waveguide laser. A polarized light pump beam at $808 \mathrm{~nm}$ generated by a tunable cw Ti:Sapphire laser (Coherent MBR 110) was focused on the sample face through a spherical convex lens $(20 \times)$. The spot radius at the input facet was about $35 \mu \mathrm{m}$. To achieve the Fabry-Perot cavity for laser emission in the waveguides, the sample input facet was coated with a thin film with high reflectivity at $1.06 \mu \mathrm{m}$ and high transmission at $808 \mathrm{~nm}$.

\section{Results and discussion}

The fs-laser inscription obviously modified the micro-structural and fluorescence properties in the filaments (fs laser focus) which correspond to the "cladding + dual-line" configuration as clearly illustrated in the fluorescence images included in Fig. 2. Fig. 2(a)-(c) shows the 2D spatial distributions of intensity, induced energy shift, and FWHM of the ${ }^{4} \mathrm{~F}_{3 / 2} \rightarrow{ }^{4} \mathrm{I}_{9 / 2}$ inter-Stark level emission line of $\mathrm{Nd}^{3+}$ ions around $940 \mathrm{~nm}$ (this line has been found to be hyper-sensitive to slight changes in the $\mathrm{Nd}^{3+}$ environment, such as strain, volume changes and disorder) $[3,7,18,25,31]$. The 3D figures shown in Fig. 2(d)-(f) are represented in order to reveal more clearly the extent of the change indicated in the $2 \mathrm{D}$ graphs. As can be seen, the fluorescence intensity image unequivocally reveals the creation of defects at the waveguide contour (i.e., partially damaged areas) as a result of the localized optical breakdown. This map indicates a good preservation of fluorescence efficiency in the waveguide region with no defects in that area. A strong change in the luminescence at the fs-laser inscription tracks is revealed by the induced spectral shift displayed in Fig. 2(b). A strong luminescence shift towards lower energies (red-shift) has been produced at the waveguide contour that is attributed to the local disorder induced in the Nd:YAG matrix as a consequence of the fs-laser induced optical damage. This is consistent with the fluorescence images of double-cladding waveguides (Type III) in
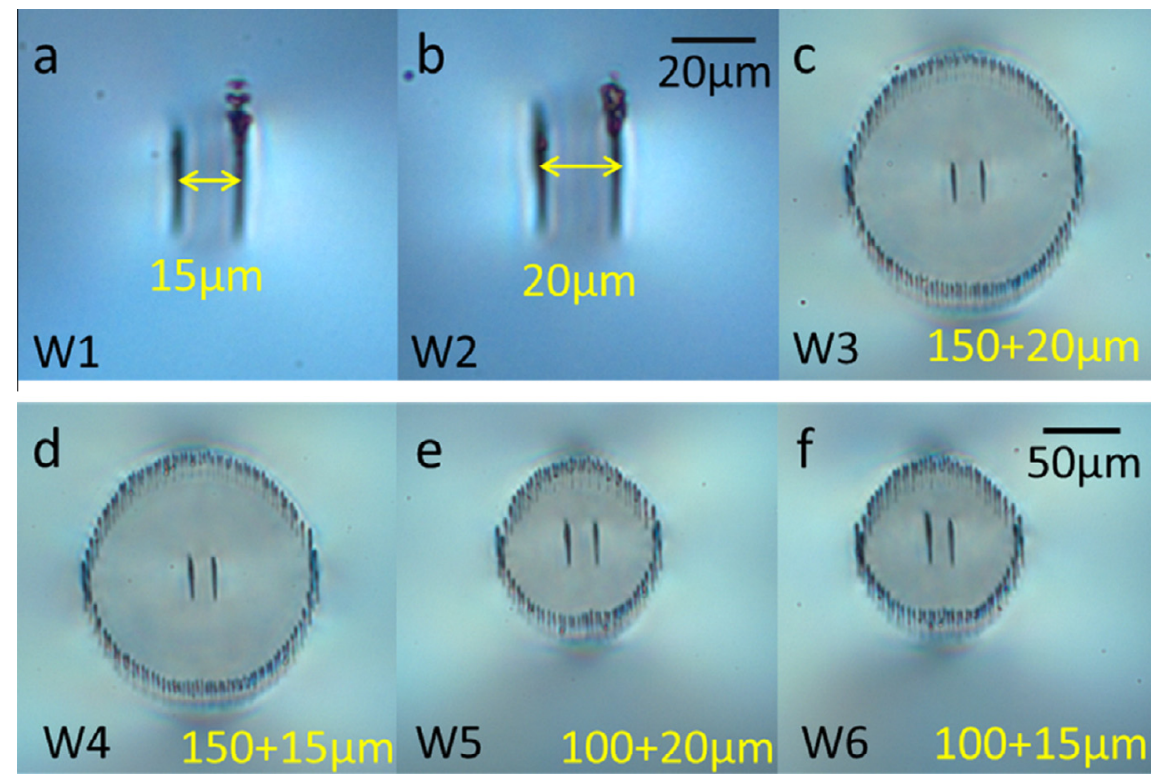

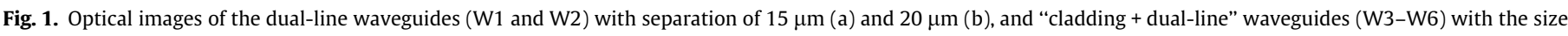
as labeled in (c)-(f). 

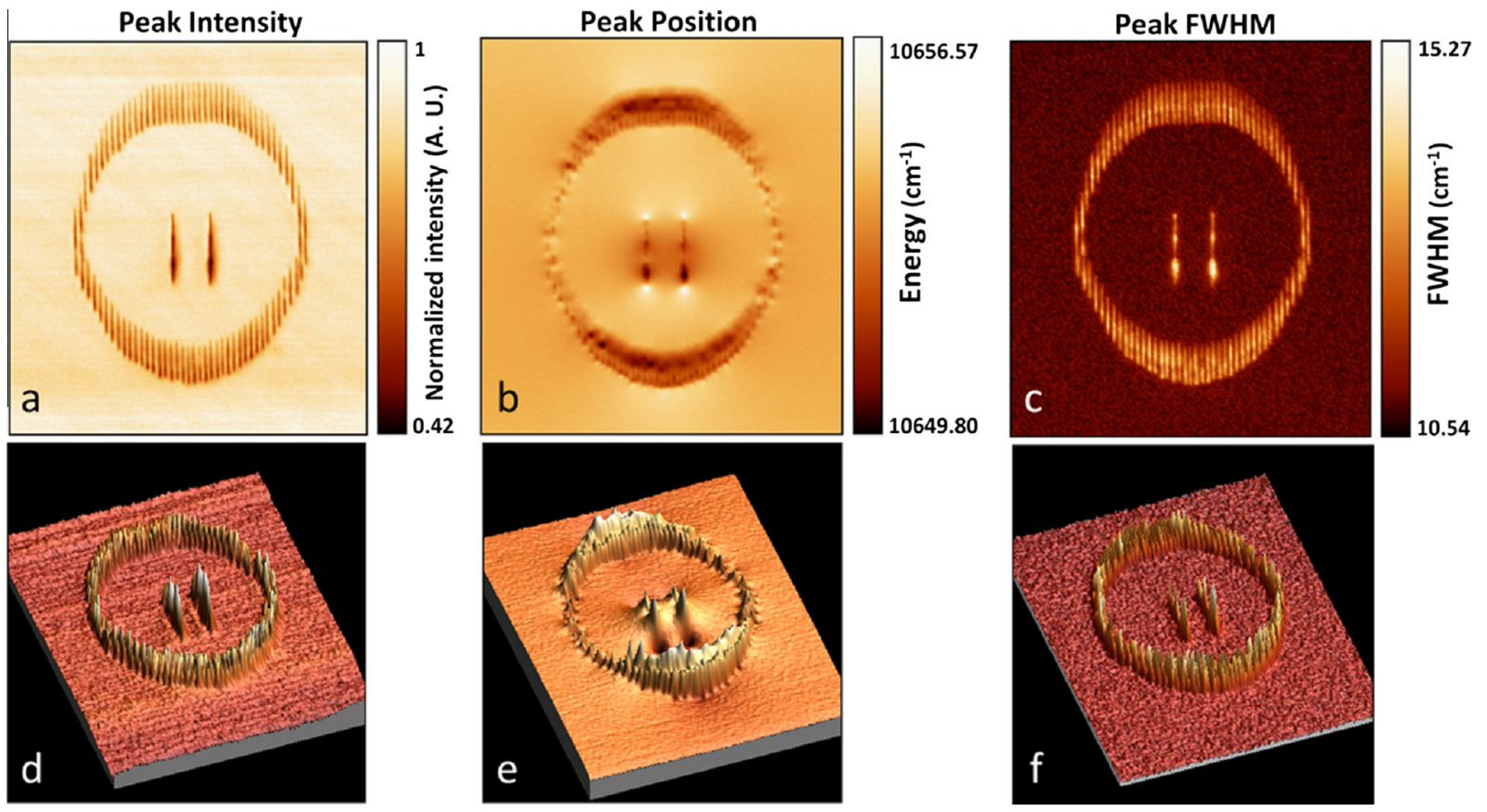

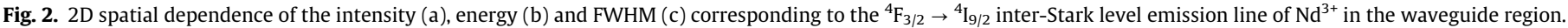
The corresponding 3D images of the spatial distribution are (d), (e) and (f).

Nd:YAG crystal [26]. Finally, the fluorescence image obtained in terms of the spectral width (FWHM) denotes a large broadening of the fluorescence lines at the waveguide profile. This could be attributed to the local disorder of the crystal matrix due to the optical breakdown induced by the focused laser. This is, indeed, in good accordance with the reduction in the signal collected by our system due to the damage or the fluorescence quenching or observed at the waveguide contour. These results suggest that structural modification mechanisms have been induced exactly at the filaments, while the fluorescence properties of $\mathrm{Nd}^{3+}$ ions is well preserved in the waveguide region.

During the fs-laser inscription process, the refractive index change at the waveguide region was caused by the induced stress and the lattice damage. The change of the refractive index in the cross section of the waveguide was measured. The distribution of $\Delta n_{v o l}$ corresponding to the contribution of the unit cell volume variation to the refractive-index change, and $\Delta n_{\text {dam }}$ corresponding to the contribution of local damage at the filaments are shown in Fig. 3(a) and (b). The refractive-index change maps were obtained through the residual strain field based on the early work on the fluorescence images, especially the spectral shift and the pressure map [3]. Under the hydrostatic pressure assumption, we calculate $\Delta n_{v o l}$ through the relative change in the unit cell volume $\Delta V / V$ as is explained in detail in a previous work [3]. As observed in Fig. 3(a), the residual stress induced by the fs-laser inscription tracks leads to both an increment of refractive index of the order of $6 \times 10^{-3}$, and a weak refractive-index reduction of the order of $-1.7 \times 10^{-3}$. As it was established in the previous works $[3,28,32]$, local lattice damage can lead to a more obvious reduction in the refractive index compared to that produced by the stress field. Fig. 3(b) shows that the reduction of refractive index is as high as $7.9 \times 10^{-2}$ exactly along the damage filaments in the crystalline network. This order of magnitude is in good agreement with that obtained in a previous work [27]. From these figures, it can be seen that an evident refractive-index change is generated at the waveguide contour as the Nd:YAG network is compressed, disordered and damaged. The map of $\Delta n_{v o l}$, indicating the stress field of the waveguide structure, reveals that a stress field with a relatively cylindrical symmetry was achieved with the external cladding, whereas the induced stress in the dual-line waveguide is highly anisotropic as reported before [3].

Fig. 4 shows the laser emission spectrum (laser intensity vs. wavelength curves) of the laser beams, which was analyzed by a spectrometer with a resolution of $0.2 \mathrm{~nm}$. The peak position remains at $1063 \mathrm{~nm}$ and the FWHM is about $0.6 \mathrm{~nm}$ The inset graphs displays the generated single mode of the laser at TM
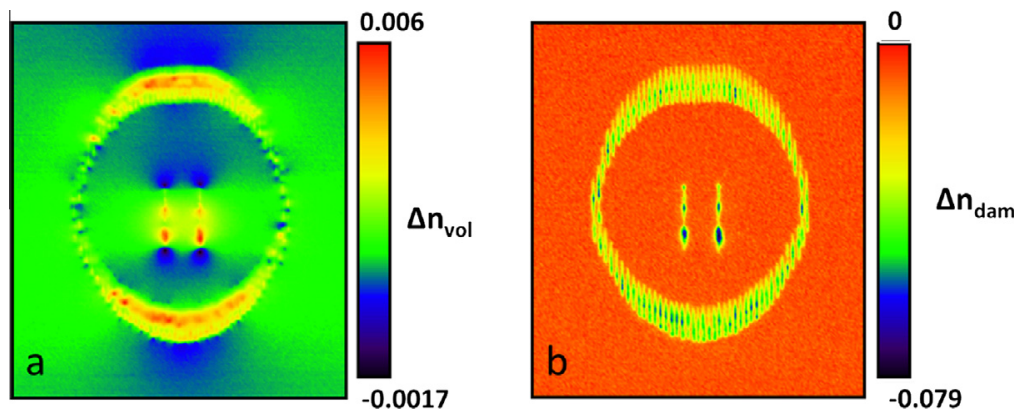

Fig. 3. Distribution of the refractive-index change in the "cladding + dual-line" waveguide area caused by (a) induced-stress, (b) lattice damage. 


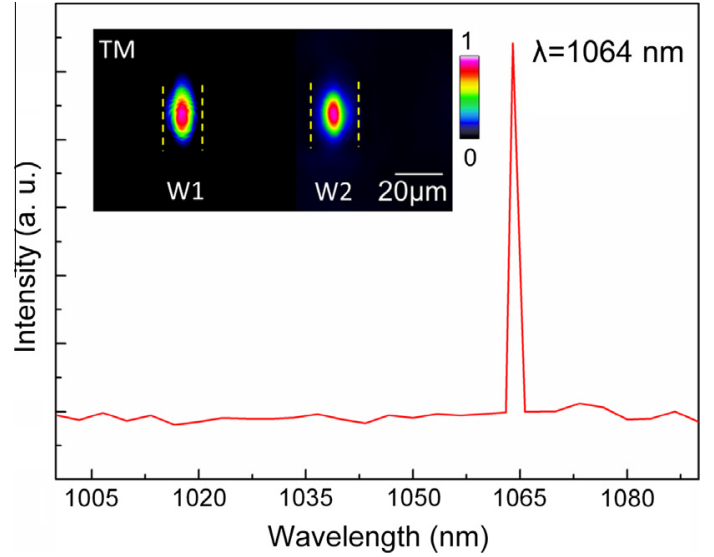

Fig. 4. Spectrum of the laser oscillation with the peak position stays at $1063 \mathrm{~nm}$ and a FWHM of $0.6 \mathrm{~nm}$. The inset graphs are the modal profiles of the laser obtained in $\mathrm{W} 1$ and $\mathrm{W} 2$ at TM polarization.

polarization in the dual-line waveguides (W1 and W2). Fig. 5(a)(h) represent the modal profiles of the waveguide laser beams from the "cladding + dual-line" waveguides (W3-W6) at TM and TE polarization. The central section of the laser modes are corresponding to the distribution of the laser oscillation generated in the dualline waveguides which certificated the good laser transmission properties at TE and TM polarizations.

In the previous work [13,14], only waveguide lasers oscillated at TM polarization were achieved in single dual-line waveguide in the cubic crystal. This is the first time that dual-line waveguides are reported to support laser oscillation at TE polarization with the modification of outer cladding structure. Laser modes differ completely from the oval modes collected in W1 and W2. The external depressed cladding structure makes a valuable contribution, modifying the induced stress field in the dual-line waveguide region, which is highly anisotropic as a consequence of the non-isotropic configuration. The fabricated outer cladding represents another stress source, whose symmetry is indeed cylindrical, making the stress field more isotropic as shown in Fig. 3(a), which is quite different from the measured-stress map in prior work [33]. Compared with the stressed field along TM polarization, the similar distribution of induced stressed field along TE polarization enables the dual-line waveguides to support the laser oscillation generation at both polarizations.

In order to compare the laser performance of dual-line waveguides in the present "cladding + dual-line" structures with that from a "dual-line" only waveguide, we utilized a rectangular aperture to filter the laser signals from the out-cladding regions to ensure the measured powers only from the inner dual-line cores. Fig. 6(a) and (b) display the output power of the laser at $1063 \mathrm{~nm}$ generated from the fs-laser inscribed dual-line waveguides with separations of $20 \mu \mathrm{m}$ and $15 \mu \mathrm{m}$, surrounded by external depressed claddings with diameters of $150 \mu \mathrm{m}$ and $100 \mu \mathrm{m}$, respectively is represented as a function of the pump power at $808 \mathrm{~nm}$. From the calculation of the experimental data with considering the transmittance and reflectivity of the optical elements

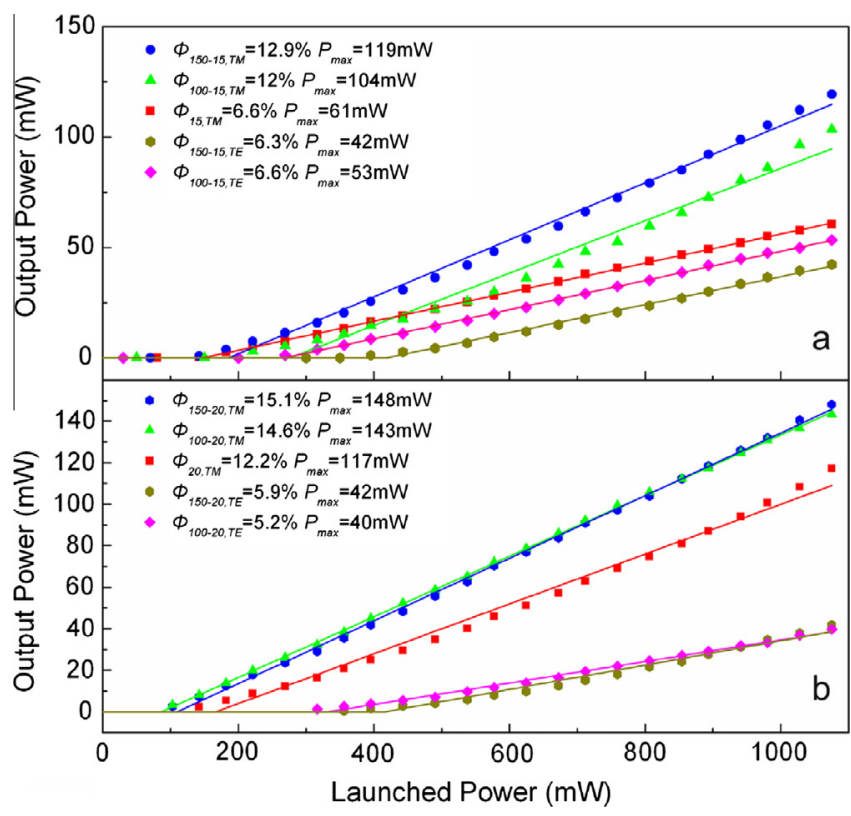

Fig. 6. The cw waveguide laser output power as a function of the launched power obtained (a) in W1, W4 and W6 with the separation of the dual-line filaments being $15 \mu \mathrm{m}$, and (b) in W2, W3 and W5 with the separation of the dual-line waveguides being $20 \mu \mathrm{m}$. The solid lines represent the linear fit of the experimental data.
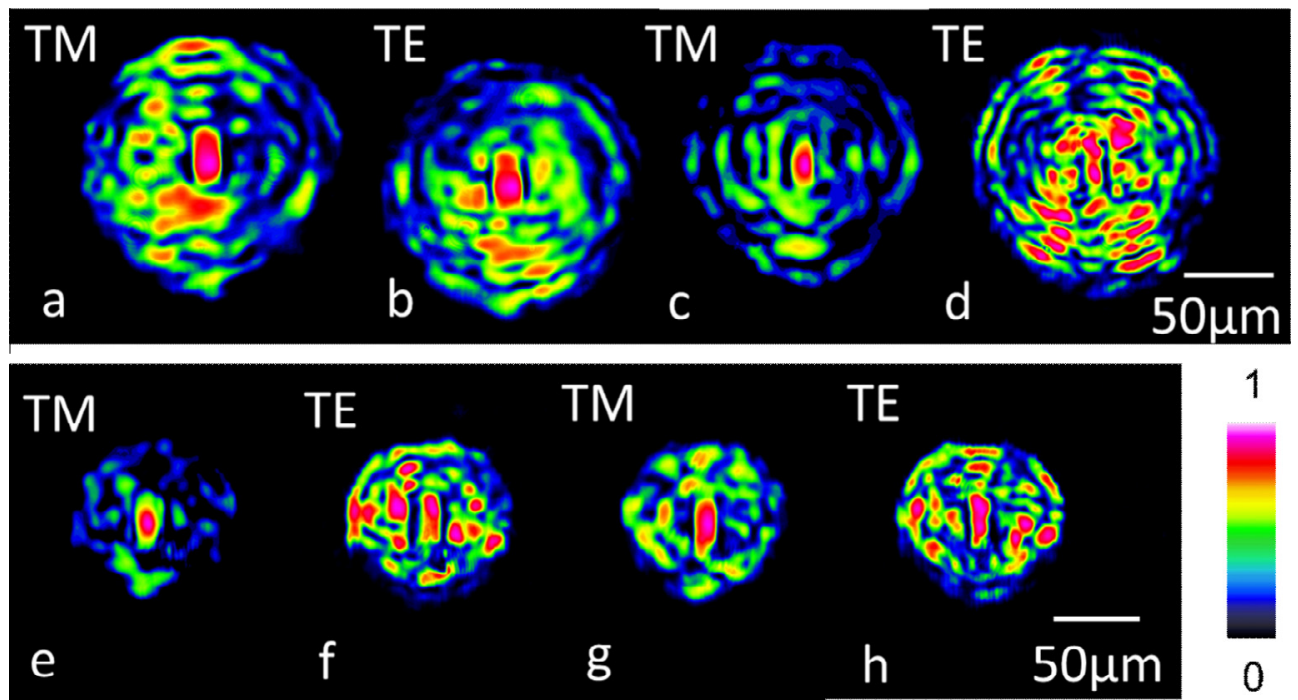

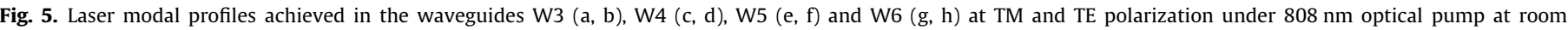
temperature. 
(e.g., microscope objective lenses), we have determined that the maximum output power for the laser oscillation at TM polarization, generated in the dual-line waveguide in W2 $\left(P_{\max }\right)$ was about $117 \mathrm{~mW}$, while the achieved slope efficiency $\left(\Phi_{20, T M}\right)$ is $12.2 \%$. By contrast, laser (at $1063 \mathrm{~nm}$ ) with a much more excellent performance was achieved in the dual-line waveguide in W3. $P_{\max }$ is about $0.15 \mathrm{~W}$ and $\Phi_{150+20, T M}$ is $15.1 \%$, which are $26.5 \%$ and $23.8 \%$ higher than those extracted from W2. In addition, the laser threshold $\left(P_{t h}\right)$ is about $102 \mathrm{~mW}$, which is $33 \%$ lower than the $P_{t h}$ of W2 $(168 \mathrm{~mW})$. For W5 and W6, the maximum output power for the laser emission at TM mode are $119 \mathrm{~mW}$ and $104 \mathrm{~mW}$, while the $P_{\max }$ of laser generated in $\mathrm{W} 1$ is about $61 \mathrm{~mW}$. Meanwhile, $\Phi_{150+15, T M}$ and $\Phi_{100+15, T M}$ are $12.9 \%$ and $12 \%$, about $90 \%$ higher than $\Phi_{15, T M}(\sim 6.6 \%)$. More importantly, laser oscillations at TE polarization were first obtained in dual-line waveguides with external cladding structures, and the maximum output power achieved is $53 \mathrm{~mW}$ with a slope efficiency of $6.6 \%$.

\section{Conclusion}

In conclusion, we have demonstrated a new photonic structure design, consisting of a tubular structure with circular cross sections of different diameters and dual-line waveguides with different separations in the center of the cladding waveguides. These structures have been fabricated in Nd:YAG crystals by femtosecond laser micro-processing. Waveguide lasers displaying a great performance have been realized in the "cladding + dual-line" structures under 808-nm optical pump. Compared to the non-isotropic stress field in the dual-line waveguides, the new design "cladding + dualline" configuration modified the stress field between the dual-line filaments, making it more isotropic. Meanwhile, waveguide lasers polarized at TM and TE orientations were achieved in the dual-line waveguides in the new configuration, which was different from the single dual-line waveguides that only supported waveguide lasers at TM polarization. CW waveguide lasers at TM polarization with higher output power $(\sim 0.15 \mathrm{~W})$, higher slope efficiency $(15.1 \%)$ and TE polarization (output power of $53 \mathrm{~mW}$ and slope efficiency of $6.6 \%$ ) were obtained when the launched power was above the oscillation threshold.

\section{Acknowledgements}

The work is supported by the Specialized Research Fund for the Doctoral Program of Higher Education of China (No. 20130131130001) and Junta de Castilla y León under Project SA086A12-2. Support from the Centro de Láseres Pulsados (CLPU) is also acknowledged.

\section{References}

[1] C. Grivas, Prog. Quantum Electron. 35 (2011) 159-239.

[2] R.R. Gattass, E. Mazur, Nat. Photon. 2 (2008) 219-225.

[3] A. Ródenas, G.A. Torchia, G. Lifante, E. Cantelar, J. Lamela, F. Jaque, L. Roso, D. Jaque, Appl. Phys. B 95 (2009) 85-96.

[4] F. He, H. Xu, Y. Cheng, J. Ni, H. Xiong, Z. Xu, K. Sugioka, K. Midorikawa, Opt. Lett. 35 (2010) 1106-1108.

[5] E. Neyra, S. Suarez, G.A. Torchia, Opt. Lett. 39 (2014) 1125-1128.

[6] Y. Liao, J. Xu, Y. Cheng, Z. Zhou, F. He, H. Sun, J. Song, X. Wang, Z. Xu, K. Sugioka, K. Midorikawa, Opt. Lett. 33 (2008) 2281-2283.

[7] F. Chen, J.R. Vázquez de Aldana, Laser Photonics Rev. 8 (2013) 251-275.

[8] B.C. Stuart, M.D. Feit, S. Herman, A.M. Rubenchik, B.W. Shore, M.D. Perry, Phys. Rev. B 53 (1996) 1749-1761.

[9] K.M. Davis, K. Miura, N. Sugimoto, K. Hirao, Opt. Lett. 21 (1996) 1729-1731.

[10] J. Thomas, M. Heinrich, P. Zeil, V. Hilbert, K. Rademaker, R. Riedel, S. Ringleb, C. Dubs, J.-P. Ruske, S. Nolte, A. Tünnermann, Phys. Status Solidi A 208 (2011) 276-283.

[11] K. Sugioka, Y. Cheng, Lab Chip 12 (2012) 3576-3589.

[12] N. Dong, J. Martinez de Mendivil, E. Cantelar, G. Lifante, J.R. Vázquez de Aldana, G. Torchia, F. Chen, D. Jaque, Appl. Phys. Lett. 98 (2011) 1111031-1111033.

[13] J. Siebenmorgen, K. Petermann, G. Huber, K. Rademaker, S. Nolte, A. Tunnermann, Appl. Phys. B 97 (2009) 251-255.

[14] C. Grivas, C. Corbari, G. Brambilla, P.G. Lagoudakis, Opt. Lett. 37 (2012) 46304632.

[15] N. Pavel, G. Salamu, F. Voicu, F. Jipa, M. Zamfirescu, T. Dascalu, Laser Phys. Lett. 10 (2013) 095802.

[16] G. Salamu, F. Jipa, M. Zamfirescu, N. Pavel, Opt. Express 22 (2014) 5177-5182.

[17] P.A. Berry, J.R. Macdonald, S.J. Beecher, S.A. MacDaniel, K.L. Schepler, A.K. Kar, Opt. Mater. Express 3 (2013) 1250-1258.

[18] A.G. Okhrimchuk, A.V. Shestakov, Opt. Lett. 30 (2005) 2248-2250.

[19] H. Liu, Y. Jia, J.R. Vázquez de Aldana, D. Jaque, F. Chen, Opt. Express 20 (2012) 18620-18629.

[20] D.G. Lancaster, S. Gross, H. Ebendorff-Heidepriem, K. Kuan, T.M. Monro, M. Ams, A. Fuerbach, M.J. Withford, Opt. Lett. 36 (2011) 1587-1589.

[21] Y. Ren, G. Brown, A. Ródenas, S. Beecher, F. Chen, A.K. Kar, Opt. Lett. 37 (2012) 3339-3341.

[22] I. Spaleniak, S. Gross, N. Jovanovic, R.J. Williams, J.S. Lawrence, M.J. Ireland, M.J. Withford, Laser Photonics Rev. 8 (2014) L1-L5.

[23] N. Dong, F. Chen, J.R. Vázquez de Aldana, Phys. Status Solidi 6 (2012) 306-308.

[24] S. Müller, T. Calmano, P.W. Metz, C. Kränkel, C. Canalias, C. Liljestrand, F. Laurell, G. Huber, Opt. Lett. 39 (2014) 1274-1277.

[25] Y. Ren, J.R. Vázquez de Aldana, F. Chen, Opt. Express 21 (2013) 11562-11567.

[26] H. Liu, F. Chen, J.R. Vázquez de Aldana, D. Jaque, Opt. Lett. 38 (2013) $3294-$ 3297.

[27] G. Palmer, S. Gross, A. Fuerbach, D.G. Lancaster, M.J. Withford, Opt. Express 21 (2013) 17413-17420.

[28] A. Okhrimchuk, V. Mezentsev, A. Shestakov, I. Bennion, Opt. Express 20 (2012) 3832-3843.

[29] D.J. Richardson, J. Nilsson, W.A. Clarkson, J. Opt. Soc. Am. B 27 (2010) B63-B92.

[30] Horcas, R. Fernández, J. M Gómez-Rodríguez, J. Colchero, J. Gómez-Herrero, A.M. Baro, Rev. Sci. Instrum. 78 (2007) 013705.

[31] A. Benayas, W.F. Silva, C. Jacinto, E. Cantelar, J. Lamela, F. Jaque, J.R. Vázquez de Aldana, G.A. Torchia, L. Roso, A.A. Kaminskii, D. Jaque, Opt. Lett. 35 (2010) 330332.

[32] J. Burghoff, S. Nolte, A. Tünnermann, Appl. Phys. A 89 (2007) (Oct. 2007) 127132.

[33] G.A. Torchia, A. Rodenas, A. Benayas, E. Cantelar, L. Roso, D. Jaque, App. Phys. Lett. 92 (2008) 111103. 